\title{
How much sleep? After the normative, the clinical sense!
}

\author{
Miguel Meira e Cruz* \\ Sleep Unit of Cardiovascular Center of University of Lisbon, Lisbon School of Medicine, Lisbon, Portugal
}

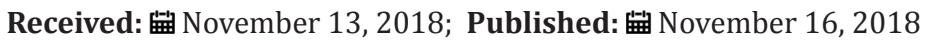

*Corresponding author: Miguel Meira e Cruz, Sleep Unit of Cardiovascular Center of University of Lisbon, Lisbon School of Medicine, Lisbon, Portugal

\section{Editorial}

Since the last three decades, we care about sleep as a relevant medical issue. We actually believe, because evidence support it, that sleep is a mediator of good health. Sleep is actually a matter of survival [1]. However, exactly how much sleep is adequate is a theme of active debate, mainly because some studies showed that either too much and too little sleep are linked to an increased risk regarding cardiovascular health and global morbidity and mortality [2]. Shorter and Longer Sleepers were stablished as normal deviations, taking account inexistent health related consequences despite shorter or longer time than the average sleep duration. Semantically we can accept this data as showing that sleep is so important that we should not jeopardize it or abuse of it. But there is much more to admire regarding the science behind these results on the adequate amount of sleep than just poetry and caution is needed because accepting non-universal facts as absolute true may sometimes really negatively impact clinical management in sleep medicine. Consensus approved that adequate amount of sleep is 7 to $9 \mathrm{~h}$ for adults and for years several health-related authorities warned about dangers associated to the non-accomplishment to that role.

Systematically, studies and research papers were published regarding sleep deprivation issues based on that reference and many patients are enrolled or not in certain therapeutic protocols because clinical criteria fit well on that statistics even though that not everything which seems, really is. Interestingly some aspects regarding natural development and adaptation were not considered in those studies which focused the change in sleep habits and sleep amount from the last century to this one when we are aware that one of the most incredible human capacity is adaptation. On the other hand, it is wise to consider sleep habits as well as sleep patterns and sleep duration as a matter of social influence and therefore influenced by the familiar context and societal circumstances.
Interestingly the paper published in 2015 by a group led by Siegel proposed that some of our old believes about human natural sleep duration could actually be misinterpreted. Light could be less powerful that temperature to reinforce circadian machinery which guide sleep and wake patterns. Thus, in a modern "natural" environment, which is intended that mimic the natural environmental of our ancestors, sleep duration and many sleep patterns were found not to be so far from that we have today in general [3]. The inexistence of evident health related problems with such studied populations within a pre-industrialized environment and the good fitness they show together with the notion of the temperature related circadian guidance allowed to the speculation that such reduced sleep time compared with recommended in modern society it is not exactly what we thought and could be misinterpreted by several bias and methodological limitations.

Furthermore it should not be forget the concept of local sleep which relates to the fact that sleep occurs not just as a global functional component but within localized areas and cellular networks and such distinctly affected areas could explain for instance the different symptoms of patients with disturbed or deprived sleep but also that not only absolute time is necessarily the most important but probably were the sleep time is happening is also of value [4]. It is possible that sum of local sleep durations could contribute to total sleep need and therefore taken into account. By contrast, maybe "local awake" should be also considered in order to recognize a homeostatic localized role which may have basic and clinical interest. Insomniacs mostly complaint of difficulty with sleep initiation, continuity or early awakening. They can complaint also of non-refreshing nocturnal sleep.

All these items are highly related to sleep duration and it is quite common that prescribers indicate some kind behavioral techniques or drugs mainly directed to the goal of sleep extension 
rather than trying to improve other sleep dimensions or consider alternative diagnostic options (for example circadian disturbance of sleep-wake cycle) at least in the very first moment of therapeutical management. On the other hand, many generalists non-specialized clinicians take normative data has a bible and enthusiastically convince patients what they should follow without considering all the aspects of individuality [5]. This may lead to inadequate management, create false perspectives and actually induce either an insomnia (if patient is suggested to sleep more than need and try to extend time in bed without sleeping) or sleep deprivation (if patient is suggested to sleep less than need). Patients with medical and sleep disorders often sleep differently from what is intended to happen in otherwise healthy people and this should be taken into account for the successful therapeutical management. Certainly, normative data is important but should not be followed without critic, without the notion of individuality and most of all should not be used without sense.

\section{References}

1. Zielinsky MR, Mckenna JT, McCarley RW (2016) Functions and Mechanisms of Sleep. AiMS Neurosci 3(1): 67-104.

2. Shen X, Wu Y, Zhang D (2016) Nightime sleep duration, 24-hour sleep duration and risk of all-cause mortality among adults: a meta-analysis of prospective cohort studies. Scientific Reports 6: 21480.

3. Yetish G, Kaplan H, Gurven M, Wood B, Pintzer H, et al. (2015) Natural Sleep and its seasonal variations in three pre-industrial societies. Curr Biol 25(21): 2862-2868.

4. Siclari F, Tononi G (2017) Local aspects of sleep and wakefulness. Curr Opin Neurobiol 44: 222-227.

5. Sateia MJ, Buysse DJ, Krystal AD, Neubauer DN, Heald JL (2017) Clinical practice guidelines for the pharmacological treatment of chronic insomnia in adults: an American Academy of Sleep Medicine clinical practice guideline. J Clin Sleep Med 13(2): 307-349.

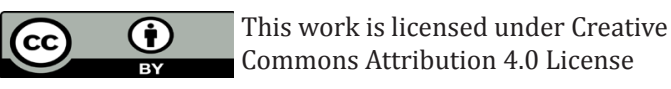

To Submit Your Article Click Here:

Submit Article

DOI: $10.32474 /$ JJNBD.2018.02.000130

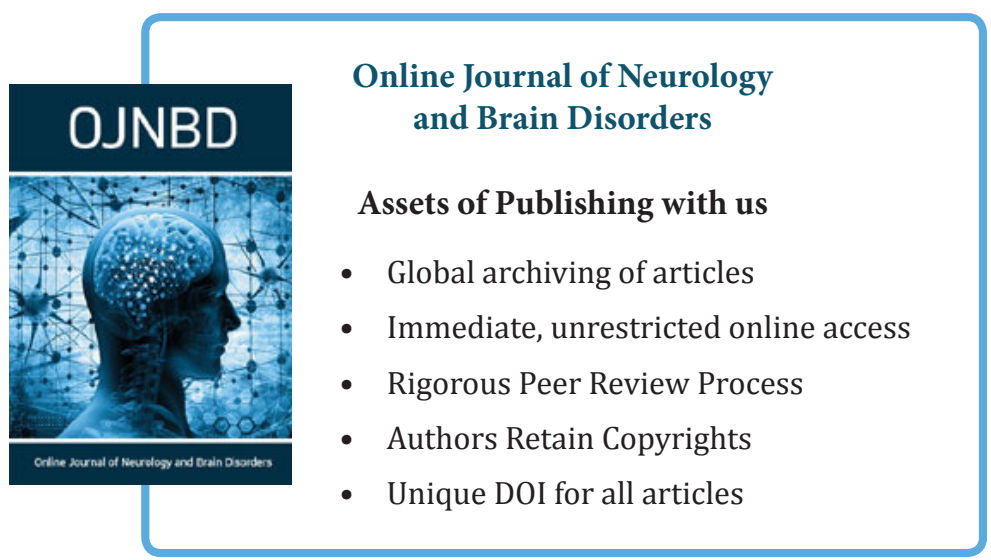

